\title{
Towards a Methodology for the Differential Analysis in Human Locomotion: A Pilot Study on the Participation of Individuals with Multiple Sclerosis
}

\author{
Huiying Yu, Thompson Sarkodie-Gyan \\ Department of Electrical Engineering, University of Texas at El Paso, Texas, USA. \\ Email: hyu@utep.edu \\ Received 2012
}

\begin{abstract}
Multiple sclerosis (MS) is an unpredictable disease of the central nervous system that can range from relatively benign to somewhat disabling to devastating, as communication between the brain and other parts of the body is disrupted. Scientists have learned a great deal about MS in recent years; yet still, its cause remains elusive. This paper intends to investigate the hypothesis that gait dynamics have meaning and may be useful in providing insight into the neural control of locomotion. It further seeks to explore the mutual interactions and influences of MS functions on gait, and vice versa, in a quantitative and robust fashion. Ground reaction forces (GRFs), muscle activities, and segmental accelerations within a gait cycle were analyzed in this study. Patterns of the signals from six relapsing-remitting multiple sclerosis (RRMS) patients were compared with the healthy subjects. This quantitative gait analysis aids to illuminate a better understanding of the mobility-related disease such as RRMS characteristics. An outcome of this study is a reproducible methodology for helping therapists make reliable and differentiable diagnosis, design a tailored therapeutic strategy, and comfortably evaluate the follow-ups on patient's functional recovery.
\end{abstract}

Keywords: Ground Reaction Forces; EMG Muscle Activities; Multiple Scelorsis; Segmental Accelerations; Kinetic and Kinematics Measurement; Fuzzy Relation Matrix; Fuzzy Similarity

\section{Introduction}

Approximately 400,000 Americans have multiple sclerosis (MS), and every week about 200 new cases are diagnosed [20]. Worldwide, MS affects around 2.5 million people: it is one of the most common chronic neurological disorders and causes of disability in young adults between 20 and 40 years of age, especially in Europe and in North America [29].

Multiple sclerosis is a demyelinating disease of the central nervous system (CNS) that can result in severe morbidity and mortality. Mobility loss and walking impairment are among the direct impact on the outcomes and the quality of life of individuals with MS. A noticeable symptom of multiple sclerosis is tremor, a rhythmic, involuntary oscillatory movement of an area of the body. Tremor caused by MS can affect the head, neck, vocal cords, trunk, and limbs, but it is most common in the arms. The two most prevalent forms of tremor in MS are postural tremor (present when holding a position against gravity) and intention tremor (present during goal-directed movement, worsening as the individual approaches a target). Tremor can be incapacitating, seriously impairing functioning and ability to perform activities of daily living. Other common motor symptoms of multiple sclerosis include foot drop, muscle weakness and/or spasticity, and altered gait rhythm. Sutliff has evaluated that maintaining mobility was ranked as one of the highest priorities among patients with MS, regardless of disease duration or disability level [27]. The loss of mobility contributes to a substantial patient burden. The statistical techniques of path analysis has shown how difficult walking significantly affects physical activity in patients with MS. Impaired mobility is associated with reductions in quality of life and activities of daily living.

Intrinsic to medical practice particularly in the evaluation of different therapeutic approaches to chronic disease, the health of the patient in association with the individual's quality of life is extremely essential. In the case of MS, a chronic debilitating disease, the preservation of the health-related quality of life becomes significant. Therefore, there is the need for measurement tools to accurately and consistently quantify the quality of life over the course of the disease. The reliable and efficient measurement of the level of impairment will assist in evaluating the variances from normal patterns through training and other compensatory strategies. To date, the evaluation of gait impairment in individuals with MS is typically performed through subjective examination by the clinician. The standardized clinical assessment tools for MS relating to gait include MS Functional Composite (MSFC) [4,12], Expanded Disability Status Scale (EDSS) [22], Disease Steps (DS) [9], MS walking scale -12 (MSWS-12) [8, 19], and other tests for balance and gait dysfunctions [1,10,15,17,18,21]. A significant problem with the subjective clinical examination is that it may be misleading due to an inability to observe the small variances from the unquantifiable impact of the observer. Therefore, clinical quantitative gait analysis must be used to provide accurate scientific evaluation through measurement of kinetic, kinematics and muscle activity.

Current clinical methods of gait analysis including the optical motion capture system are time and labor intensive and involve extensive post-hoc data analysis [2,6,11]. These limitations 
reduce access to gait analysis and exclude direct application of the patient's gait data to rehabilitative interventions in real-time. In contrast, inertial sensor systems with its properties of small size, light weight, lower cost, direct measurement, and their unobtrusive effect on the human is becoming an important methodology in biomechanics and clinical applications. Liu et al. used the inertial sensor system (gyroscopes and accelerometers) to detect the gait phases [16]. However, quantitative gait analysis using inertial sensor system in individuals with MS has not yet attracted any relevance in clinical application. In fact, the most important symptoms of MS include muscle weakness and spasticity and hence the essence of evaluating the muscle activities in individuals with MS.

This study involved the collection of pilot data in MS patients with mild to moderate gait difficulty. The fusion of the acquired multiple sensor data and the fuzzy inferential reasoning algorithm were applied to quantify and differentiate the kinetic, kinematics and electromyographic data for correlation with each subject's physiological states, health conditions, and the underlying mechanisms of disability, if any.

\section{Data Acquisition and Signal Processing}

\subsection{Participants}

Subjects were recruited from the local community. All subjects provided informed consent prior to participation, and the Institutional Review Board at the University of Texas at El Paso (UTEP) approved the study protocol.

Six patients were recruited, and their respective primary care physicians made the diagnosis, and classified them as relapsing-remitting multiple sclerosis (RRMS). Each patient had subjective experience with reduced walking ability, from mild to moderate levels; they had an expanded disability status scale (EDSS) score of 6.0 or less. Table 1 illustrates a short description of each RRMS patient, and five of them had no others (non MS-related) conditions affecting walking ability. Twenty-two healthy subjects were recruited as control group. Twelve male subjects with age $22.8 \pm 2.12$ years old, height $1.70 \pm 7.37 \mathrm{~m}$, weight $68.5 \pm 5.56 \mathrm{~kg}$, BMI $23.9 \pm 2.05 \mathrm{~kg} / \mathrm{m}^{2}$, and treadmill walking speed $0.99 \pm 0.07 \mathrm{~m} / \mathrm{s}$; ten female subjects with age $23 \pm 2.31$ years old, height $1.65 \pm 4.80 \mathrm{~m}$, weight $58.4 \pm 6.47 \mathrm{~kg}$, BMI $21.9 \pm 1.74 \mathrm{~kg} / \mathrm{m}^{2}$, and treadmill walking speed $0.96 \pm 0.09 \mathrm{~m} / \mathrm{s}$.

\subsection{Equipments}

- Instrumented treadmill
GRFs were measured using an instrumented treadmill (Bertec ${ }^{\circledR}$ Corporation,USA). The treadmill is a dual-belt force sensing component, records the consecutive left and right GRFs in three dimensions: mediolateral $(F x)$, anterioposterior $(F y)$, and vertical $(F z)$. GRF is a 'reflection of the total mass-timesacceleration product of all body segments and therefore represents the total of all net muscle and gravitational forceactions at each instant of time over the stance phase' [28]. Therefore, accurate measurement on the GRF can provide significant information for the study of normal and pathologic gait.

- Surface Electromyography (sEMG)

Delsys surface EMG with its Myomonior ${ }^{\circledR}$ (Delsys Inc., MA, U.S.A.) was used to measure the muscle activities on the lower extremity. The surface electrodes were placed on the skin overlying the muscle, and therefore can noninvasively measure the muscle activity in dynamic motion such as walking and running. Surface EMG (sEMG) signals are often used as a diagnostic tool for identifying neuromuscular diseases as well as control signals for electronic bio-devices such as prosthetic hands, arms, and lower limbs [24].

- Accelerometers

Tri-axial ADXL330 iMEMS ${ }^{\circledR}$ accelerometers from Analog Device Inc. were used to measure the accelerations directly at the point of attachment. Advantages of using accelerometers over other biomechanical devices primarily are expressed by their miniature size, hence offer limited restrictions of their anatomical placement and provide minimal impediment to movement. Direct and continuing measurement of accelerations therefore, can be used as wearable biosensors to measure gait parameters in real world situations without the limitations inherent in immobile laboratory approaches such as optical motion capture systems [25].

\subsection{Experimental Procedure}

The complete sensor placements including force sensors, surface EMG electrodes and tri-axial accelerometers are illustrated in Figure 1. Dynamic left and right GRFs in three dimensions were measured independently. The following eight muscles on both extremities (totally 16 EMG electrode placements) were recorded: Soleus (Sol), Tibialis Anterior (TA), Gastrocnemius Lateralis $(L G)$, Vastus Lateralis $(V L)$, Rectus Femoris $(R F)$, Biceps Femoris $(B F)$, Gluteus Medius (Gmed), and Erector Spinae (ES). Eight Tri-axial accelerometers were placed on the lower body segments: foot $\left(F t_{x}, F t_{y}, F t_{z}\right)$, shank $\left(S k_{x}, S k_{y}, S k_{z}\right)$, thigh $\left(T h_{x}, T h_{y}, T h_{z}\right)$, and hip $\left(H i p_{x}, H i p_{y}, H i p_{z}\right)$.

Table 1. MS patient's characteristics.

\begin{tabular}{|c|c|c|c|c|c|c|}
\hline $\begin{array}{c}\text { MS } \\
\text { Patient }\end{array}$ & $\begin{array}{l}\text { Sex } \\
M / F\end{array}$ & $\begin{array}{l}\text { Age } \\
\text { years }\end{array}$ & $\begin{array}{c}\text { BMI } \\
\left(\mathrm{kg} / \mathrm{m}^{2}\right)\end{array}$ & $\begin{array}{c}\text { Speed } \\
(\mathrm{m} / \mathrm{s})\end{array}$ & Observed gait impairment & Medicine \\
\hline 1 & $\mathrm{~F}$ & 55 & 29.4 & 0.65 & Wobbly gait with no aids & Interferon beta \\
\hline 2 & $\mathrm{~F}$ & 40 & 22.5 & 0.65 & Mild left foot drop, occasional tremor, walking with no aids & Interferon beta \\
\hline 3 & M & 62 & 29.6 & 0.15 & Walking with unilateral cane, foot drop & Interferon beta \\
\hline 4 & M & 37 & 39.9 & 0.35 & Limp gait, walking with unilateral cane, foot drop & Interferon beta; Desyrel \\
\hline 5 & M & 45 & 39.2 & 0.75 & No visible gait abnormality, walking with no aids & Copaxone \\
\hline 6 & M & 28 & 21.8 & 0.50 & Wobbly gait with no aids, right leg postural tremor & Naltrexon \\
\hline
\end{tabular}

BMI - Body Mass Index $\left(\mathrm{kg} / \mathrm{m}^{2}\right) \quad$ RRMS - Relapsing Remitting Multiple Sclerosis 


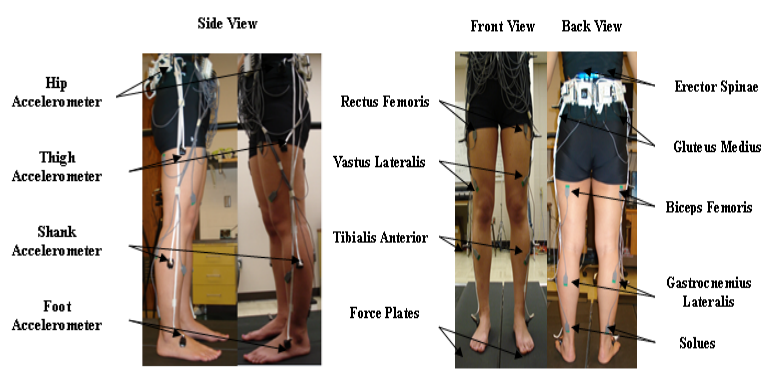

Figure 1. Multiple Sensors Placements for Data Acquisition.

Each subject wore running shorts and comfortable T-shirt during the experiment. Subjects were allowed to become familiar with the walking track on the treadmill before conducting the experiment. For patients, partial body-weight support (harness) fixed on the instrumented treadmill was applied for safety purposes. The speed of the treadmill was incrementally increased or decreased to determine the participant's comfortable walking speed. Every subject was instructed to walk continuously for three minutes on the treadmill.

The LabView software was used for the acquisition of accelerations and GRFs data at sampling frequency of $100 \mathrm{~Hz}$. EMG data was acquired at sampling frequency of $1000 \mathrm{~Hz}$ using Delsys EMGWorks acquisition software. The synchronization between the acquired data was achieved by Delsys EMG trigger module. Therefore, the GRFs, EMGs, and acceleration signals were collected simultaneously.

\subsection{Data Processing}

White noise was observed in the force data due to the vibrations and the motion artifact during the walking process on the treadmill. The GRF data were smoothened using second order Butterworth low pass filter with cut-off frequency of $20 \mathrm{~Hz}$. The second order Butterworth low pass filter with cutoff frequency of $6 \mathrm{~Hz}$ was applied to reduce the noise and improve the resolution of the acceleration data.

Since unprocessed EMG data or raw EMG data cannot be used for quantitative comparison between subjects, the EMG raw data were filtered by: Band Pass Filtering (second order Butterworth band-pass filter with a frequency of 20-200 Hz), Full Wave Rectification, and Linear Envelope (second order Butterworth low pass digital filter with a cutoff frequency of 7 $\mathrm{Hz})$.

\subsection{Fuzzy Relation Matrix and Fuzzy Similarity}

In this study, a cluster of data relating to the kinematics, kinetics and muscle activities was acquired using an array of inertial sensors (accelerometers), instrumented treadmill and electromyographic device during normal walking tasks. These data were treated as aggregate information granules that enabled the efficient partition of input space and more rapid analysis. This means that we dealt with the relationships of the kinematic, kinetic and muscle activity functions. The relationships depict the attributive features of the human movement and are expressed in an implication table giving rise to a fuzzy relational matrix, established between the dynamic activities during the walking tasks. Therefore, we established granulated information between 1) the acquired kinetic and kinematic data and the gait phases; and 2) the acquired muscle activity data and the gait phases. Since data are usually represented in a tabular form, it is also easy to consider the table as a matrix. Hence, a fuzzy relational matrix was used as the expression of the strength of association or interaction amongst the elements of the gait functions, and it elucidates a rule base that is used to provide a model, a model of a feature matrix.

GRF data were normalized by individual body weight, whereas EMG and acceleration data were normalized by the maximum averaged values. Normalized Data were then aver- aged from 100 strides (100 gait cycles). Averaged data within a gait cycle with seven functional gait phases were extracted using the vertical GRFs [11]. The seven functional gait phases as illustrated in Figure 2 are: Loading Response (LR), Mid- stance (MST), Terminal Stance (TST), Preswing (PSW), Initial Swing (ISW), Midswing (MSW), and Terminal Swing (TSW) [28]. The mean values of the data in the seven gait phases are represented in a matrix form, fuzzy relational matrix.

Three types of fuzzy relational matrices were constructed using the methods in [11]. The relational matrix between GRFs $\{F x, F y, F z\}$ and the 7 gait phases $\left\{P_{1}, P_{2}, P_{3}, P_{4}, P_{5}, P_{6}, P_{7}\right\}$; EMG $\left\{\begin{array}{llllllll}\text { Sol TA } & L G & V L & R F & B F & G m e d & E S\}\end{array}\right\}$ and the seven gait phases; the accelerations

$\left\{F t_{x}, F t_{y}, \quad F t_{z}, S k_{x}, S k_{y}, S k_{z}, T h_{x}, T h_{y}, T h_{z}, H i p_{x}, H i p_{y}, H i p_{z}\right\}$ and the seven gait phases.

Fuzzy similarity of each parameter was calculated using the equation (3) defined in [11]. This methodology involving the acquisition of the kinematics, kinetics and electromyographic data of human gait enable the understanding of the neurophysiology/biomechanics of gait for augmenting objective measurements of mobility and functional status. This methodology may be applied for the efficient and reliable analysis of human gait dynamics at a level that quantifies variations in MS from morphometrically adjusted normal.

\section{Results}

The GRFs, the EMG muscle activities, and the accelerations within a gait cycle were analyzed in this study. Patterns of the signals from six RRMS patients were compared with the healthy subjects. The pathologic characteristics of multiple sclerosis were concluded and the intelligent algorithm provided a quantitative analysis for the clinical diagnosis for MS patients.

In Figure 3(a), the normalizations of the 3-D GRFs (GRFs in \% $\mathrm{BW}$ ) with respect to the gait cycle were performed for both healthy and MS patients. Figure 3(b) illustrates the fuzzy simi- larities between the GRFs of the MS patients and the $\begin{array}{lllllll}\mathrm{h} & \mathrm{e} & \mathrm{a} & \mathrm{l} & \mathrm{t} & \mathrm{h} & \mathrm{y}\end{array}$

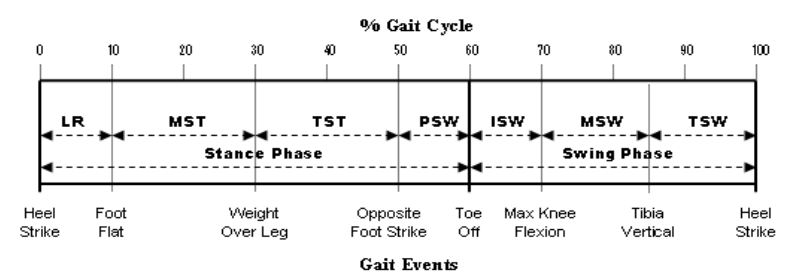


Figure 2. Seven gait phases using \% gait cycle and transitional gait events.

subjects in three dimensions. This similarity ranges between 0 and 1 , and it represents the strength of the association between the GRFs of the healthy subjects and MS patients within the respective gait phase.

Eight normalized EMG data within a gait cycle were illustrated in Figure 4(a). The Figure presents the gait pattern of the healthy subject as an asymptote to the variances in the gait patterns of the individual patients. Figure 4(b) illustrates the grade of similarities between the eight EMGs of the MS patients and the healthy subjects within the seven gait phases.

Normalized 3-D acceleration data on four body segments (foot, shank, thigh, and hip) within a gait cycle are shown in Figure 5(a). Figure 5(b) represents the fuzzy similarities of accelerations of the MS patients within the seven gait phases.

\section{Discussion}

The self-selected walking speeds of the MS patients were generally lower as compared with those of the healthy subjects (range $0.9 \sim 1.2 \mathrm{~m} / \mathrm{s}$ ). The effects can be observed from the
GRFs in all dimensions, i.e. the increased stance phase/double stance phase, decreased swing phase. The GRF pattern has simply shown an estimated disease-severity of the individual, RRMS-3 subject for instance was found with more severe symptoms along all six MS patients. This may also be due to the age of this patient (62 years old) with other gait-related disorders such as diabetes (information not shown in methods).

A previous research result suggested that the GRFs varied significantly between the MS patients and the healthy subjects in both vertical and anterior-posterior directions but showed no differences in the mediolateral direction [23, 30]. However this study found that the vertical GRF of the MS patients did not depict the reference $\mathrm{M}$-shaped pattern, and the lower magnitude of the anterior-posterior forces appear in most of the MS patients.

The result illustrated in Figure 3(a) suggests a common feature of MS patients in the vertical GRF, the magnitude of the first peak is excessively high in the early stance phase, whereas that of the second peak is moderate in the terminal stance. In normal gait the magnitude of the two peaks are approximately

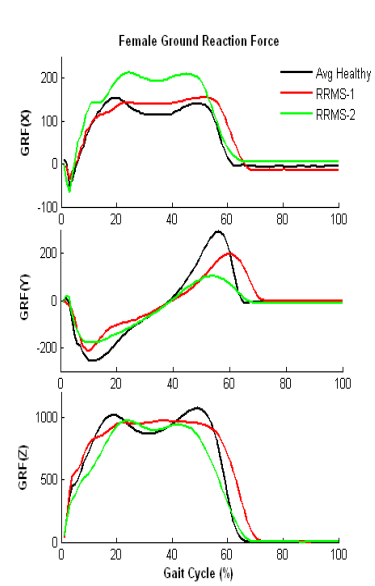

(a)

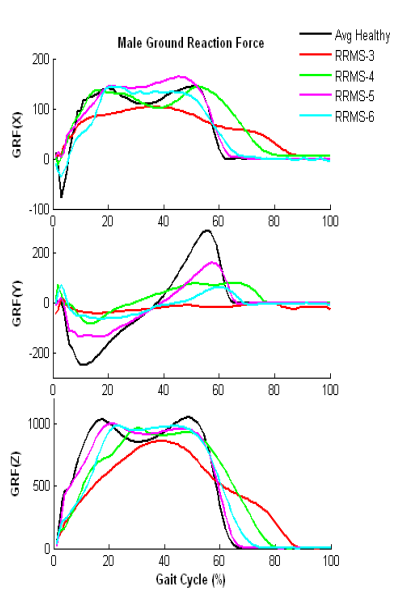

)

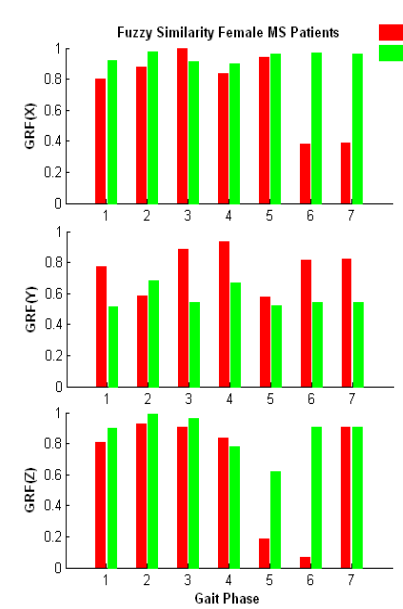

(b)

Figure 3. Ground Reaction Force Pattern within a Gait Cycle and Fuzzy Similarities of the Seven Gait Phases.
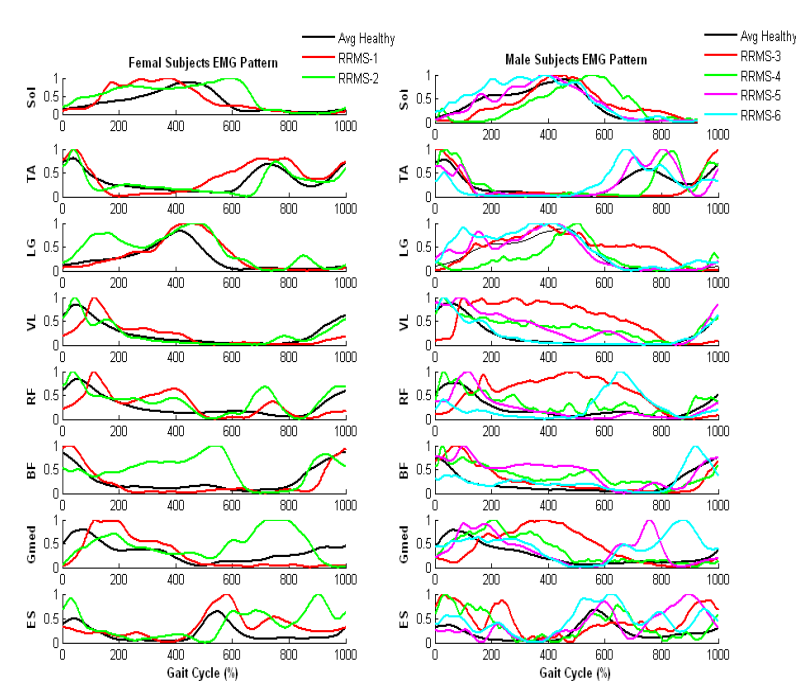

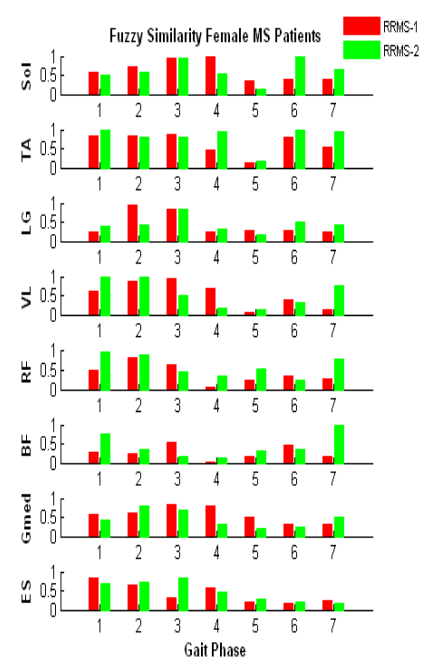

2 Funzy Similarity Male MS Patients 2 $\leqslant 05\left[\begin{array}{ll}1 \\ 0\end{array}\right.$ 305

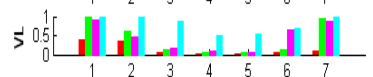
妾 狊 0.5 :

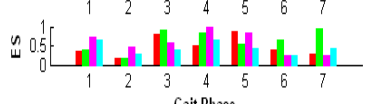


(a)

(b)

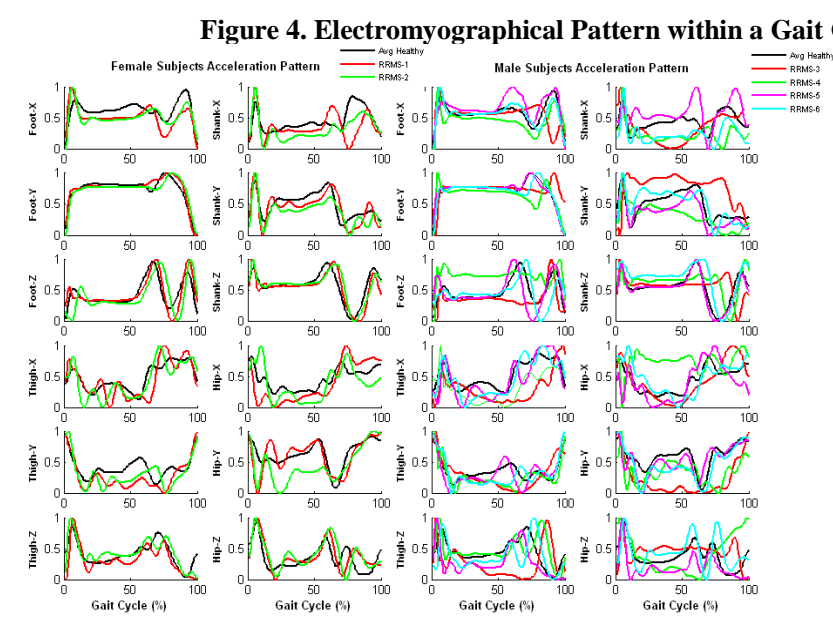

(a)

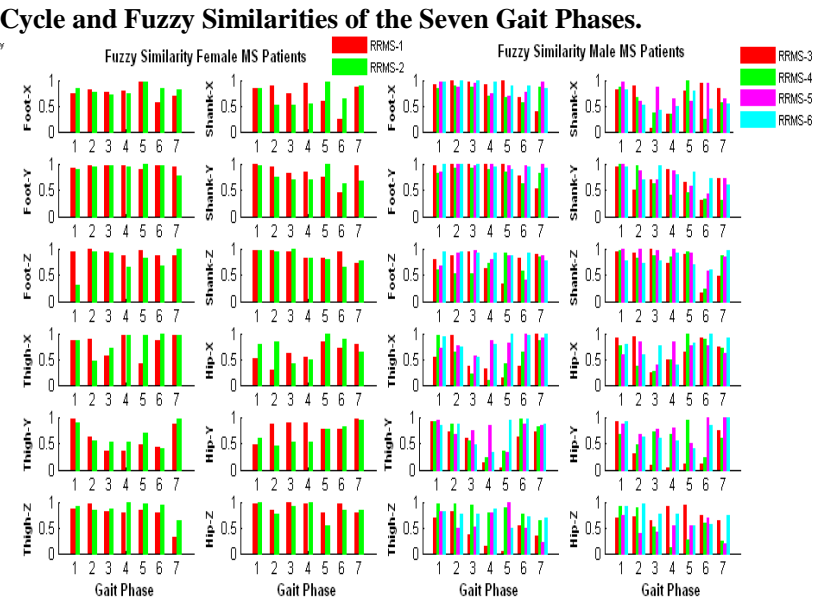

(b)

Figure 5. Acceleration Pattern within a Gait Cycle and Fuzzy Similarities of the Seven Gait Phases.

equal in which the M-shape force curve illustrates the weight transfer from the heel to the mid-foot and the mid-foot to the ball of the foot for push-off. The fact that the vertical GRF exceeds body weight in the terminal stance (i.e. second peak of the vertical GRF) indicates that the body weight is being supported and some propulsion is generated successfully. On the other hand, the low propulsive force is generated in the MS patient, and the greater impairments (much more reduced second peak or sometimes even less than the body weight) indicated a more severely affected MS patient (RRMS-3). By comparing the magnitude of the anterior- posterior force of the healthy subjects, the MS subjects revealed much lower magnitude of the force.

This study has also found different patterns in mediolateral GRF. Apart from the RRMS-3 patient, the higher magnitudes of the mediolateral force were found in all other five patients during the pre- and terminal stance phase (i.e. single support phase). The mediolateral force in normal gait is of lower magnitude in most situations and relates to balance during walking. The higher magnitude of the mediolateral force with MS patients indicates that more effort was applied to keep the balance, especially during the single support phase.

Figure 4(a) illustrates the eight muscle activities of both the healthy and MS subjects, respectively. The Soleus and Gastrocnemius muscles are ankle plantarflexors, and designed to stabilize the foot and knee during the stance phase of the gait. However, most of the MS subjects exhibited long-latency periods with increased activities on the Soleus and Gastrocnemius in the stance phase.

The Tibialis Anterior (TA) is an ankle dorsiflexor and most active at heel strike and prevents "foot-slap". The behavior of the TA in this study was significantly greater during the initial contact and during the toe-off. This increased EMG activity that was apparent in the ankle dorsiflexor muscles in the MS subjects are considered the mechanism for counteract-balancing the deficits. The exhibited behavior may illustrate implications relating to fatigue and spasticity [13]. In addition, the TA activity of most of the MS patients was significantly greater during the pre-swing phase and the early swing phase. This activity is supposed to assist the toes in clearing the floor for the push-off into swing.

Vastus Lateralis (VL) and Rectus Femoris (RF) muscles depict the knee extensors/hip flexors and the muscles of quadriceps group. Most of the MS subjects showed intense and prolonged action of the VL and RF from the midstance through to the initial swing. Extensor spasticity of the legs, particularly of the quadriceps, might be considered advantageous for standing, walking and particularly transferring, as it may compensate for muscular weakness. Clinical research has suggested that some structural changes of spastic muscles and of connective tissues develop over periods of weeks to months in MS patients, leading to changes in mechanical properties in the leg extensors. This finding may therefore be due to inadequate management of spasticity which leads to such structural changes of muscles with subsequent functional impairment [14].

Biceps Femoris (BF) muscle is the lateral hamstring, which is activated to accelerate the knee toward flexion and the hip toward extension during swing in normal gait. This was found in the prolonged and increased muscle activity of BF in the stance phase, particularly in the single-support phase, whereas a delay and reduced muscle activity of BF in the midswing through terminal swing phase. These findings suggest that the prolonged stance activity of the upper leg muscles, particularly those knee and hip extensors, could be related to a more general neuromuscular strategy that serves to supply additional support. Evidence for the use of this strategy was found in hemiparetic stroke from a recent muscle actuated simulation of hemiparetic gait by Higginson, who showed that co-contraction of the upper leg muscles during midstance can be used to enhance knee stability [7]. It is suggested that this strategy is used to compensate BF weakness in the swing phase. These spastic upper leg extensors may in turn cause delayed Gluteus medius (Gmed) in the loading response. Gluteus medius is the hip abductor and pelvic stabilizer. Figure 4(a) shows the MS subjects with greater activity of Gmed in the midstance and midswing phases to support the body weight with single leg 
over the ground.

Erector Spinae (ES) or lower back muscle monitors the paraspinal activity of the trunk movement and stabilization. The EMG activation level of the ES was found to be greater in the MS than in healthy subjects at the swing-stance and stance-swing transitions, which is likely to be a compensatory factor in the instability during phase transition, as well as serving to reduce the risk of falling.

The use of accelerometers is an important alternative approach for the acquisition of kinematic data in gait analysis techniques. Accelerometers placed on the foot, shank, thigh, and hip reveal both temporal aspects of the gait cycle and acceleration forces at key events in the cycle, such as heel strike, toe-off, and events during swing phase. Study of the acceleration/deceleration patterns in MS patients can further confirm the muscle spasticity or muscle weakness and physiological tremor in the individual gait. There were significant different patterns of acceleration in MS subjects as compared with the healthy subjects, especially in the anterioposterior and mediolateral directions.

In the mediolateral direction, there was a greater acceleration occurring in the initial contact during the loading response (the first $10 \%$ of the gait cycle) in the MS subjects. It is thought to be the compensatory mechanism for the stability of the ankle and knee. The acceleration in the following gait cycle on that direction was generally reduced in most of the MS subjects, an expected occurrence with respect to the slow working speeds.

In the anterior-posterior direction, a sharp acceleration in the initial contact was found in most of the MS subjects, which depicts an indication of faster heel-to-toe force transfers at that time, especially for patients with foot-drop. On the foot acceleration pattern in this direction, there were either moderate deceleration at toe-off observed in RRMS-1 and RRMS-2, or faster deceleration followed by decreased acceleration at the beginning of the initial swing seen in RRMS-3 RRMS-6. This is an indication of an inadequate toe clearance in the MS subjects, resulting in reduced forward progression of the limb with push off into swing.

The histogram of Figure 3(b) illustrates a relational mapping between the GRFs of the subject with respect to the seven gait phases, for both male and female subjects. The normalized " 1 " is an asymptote of the GRFs of a healthy patient and is used as the reference pattern. The comparative relationship between the healthy subject and the MS patients illustrates a functional association between the elements of the GRFs and the gait phases. The same illustrations shown in Figures 4(b) and 5(b) for the muscles activities and acceleration respectively. The asymptotes depicted in the histogram illustrate a direct grade of aggregation or association between the physiologic data of the MS patient with respect to those of the healthy subject. This strength of aggregation is a functional relationship that clearly illustrates an objective and differential assessment of the efficacy and outcomes of rehabilitation therapies and practices. This will also enable the further development of precise methods for measuring impairments and disabilities.

\section{Conclusions}

This study offers a narrative description of the dynamic phasic patterns of GRFs, muscle activities, and accelerations in the body segments in the MS patients. The study enables the analy- sis of multiple components in three-dimensional plane for the reliable differentiation of pathological and/or compensatory functional impairments in MS and in normal gait patterns. The fuzzy inferential reasoning algorithm provides the understanding of the dynamic patterns of these components. In real-time data acquisition and pattern analysis, this intelligent methodology will serve as a self-motivated tool for the individual subject during treatment. The technology may help therapists in choosing a tailored therapeutic strategy for the individual, predict responses to treatment, or determine if maximal recovery has occurred.

\section{Acknowledgements}

The authors wish to thank the Stern Foundation for providing the funds for this research work.

\section{REFERENCES}

[1] Albrecht H., Wotzel C., Erasmus L.P., Kleinpeter M., Konig N., Pollmann W., "Day-to-day variability of maximum walking distance in MS patients can mislead to relevant changes in the Expanded Disability Status Scale (EDSS): average walking speed is a more constant parameter”, Mult Scler. 2001;7(2):105-109

[2] Cappozzo A., Catani F., Leardini A., Benedetti M.G., Croce UD., "Position and orientation in space of bones during movement: experimental artifacts.” Clin Biomech,1996; 11(2):90-100

[3] De Luca C.J. "The use of surface electromyography in biomechanics” Journal of Applied Biomechanics, 1997; 13(2): 135-163

[4] Fischer J.S., Rudick R.A., Cutter G.R., Reingold S.C., "The Multiple Sclerosis Functional Composite measure (MSFC): an integrated approach to MS clinical outcome assessment”, Mult Scler August 1999; 5:244-250

[5] Giansanti D., Macellari V., and Maccioni G. (2005) "The development and test of a device for the reconstruction of 3-D position and orientation by means of a kinematic sensor assembly with rate gyroscopes and accelerometers” IEEE Trans Biomed Eng, vol. 52, no. 7, pp. 1271-1277

[6] Hausdorff J.M., "Gait variability: methods, modeling and meaning” Journal of NeuroEngineering and Rehabilitation, 2005; 2:19-27

[7] Higginson J.S., Zajac F.E., Neptune R.R., Kautz S.A., Delp S.L., "Muscle contributions to support during gait in an individual with post-stroke hemiparesis”. J Biomech 2005

[8] Hobart J.C., Riazi A., Lamping D.L., Fitzpatrick R., Thompson A.J., "Measuring the impact of MS on walking ability. The 12-item MS Walking Scale (MSWS-12)”, Neurology. 2003;60(1):31-36

[9] Hohol M.J., Orav E.J., Weiner H.L., "Disease Steps in multiple sclerosis: A simple approach to evaluate disease progression", Neurology February, 1995;45(2):251-255

[10] Hoogervorst E.L., van Winsen L.M., Eikelenboom M.J., Kalkers N.F., Untdehaag B.M., Polman C.H., "Comparisons of patient self-report, neurologic examination, and functional impairment in MS”, Neurology. 2001; 56(7):934-937

[11] Huiying Yu, Murad Alaqtash, Eric Spier, Thompson Sarkodie-Gyan, "Analysis of muscle activity during gait cycle using fuzzy rule-based reasoning”, Elsevier Journal of Measurement, April, 2010; 43(9):1106-1114

[12] Kaufman M., Moyer D., Norton J., "The significant change for the Timed 25-Foot Walk in Multiple Sclerosis Functional Composite”, Mult Scler. 2000;6(4):286-290 
[13] Kelleher K.J., Spence W.D., Solomonidis S.E., Apatsidis D. “The characterization of gait patterns with multiple sclerosis”. Disabil Rehabil. 2010; 32(15): 1242-50

[14] Kesselring J., "Multiple Sclerosis”. Cambridge University Press, 1997

[15] Legters K., Whitney S.L., Porter R., Buczek F., “The relationship between the Activities-specific Balance Confidence Scale and the Dynamic Gait Index in peripheral vestibular dysfunction”, Physiotherapy Research International. 2005;10(1):10-22

[16] Liu T., Inoue Y., Shibata K., "Development of a wearable sensor system for quantitative gait analysis”, Measurement. 2009; 42(7):978-988

[17] Marchetti G.F., Whitney S.L., "Construction and Validation of the 4-Item Dynamic Gait Index”, Physical Therapy. 2006;86(12):1651-1660

[18] McConvey J., Bennett S.E., "Reliability of the Dynamic Gait Index in Individuals with multiple sclerosis", Archives of Physical Medicine and Rehabilitation. 2005; 86:130-133

[19] McGuigan C., Hutchinson M., "Confirming the validity and responsiveness of the Multiple Sclerosis Walking Scale-12 (MSWS-12)”, Neurology. 2004;62(11):2103-2105

[20] National Institute of Neurological Disorders and Stroke (NINDS). (2006a, last updated January). NINDS Multiple Sclerosis Information Page, 2006

[21] Nieuwenhuis M.M., Tongeren H.V., Sorensen P.S., Ravnborg M., "The Six Spot Step Test: a new measurement for walking ability in multiple sclerosis”, Mult Scler. 2006;12(4):495-500

[22] Noseworthy J.H., "Clinical scoring methods for multiple sclerosis”, Ann Neurol. 1994; 36 Suppl:S80-5
[23] Pringle D., Seger A.M., and Ponichtera-Mulcare J., "Locomotive function in individuals with multiple sclerosis”, Biomedical Engineering Conference, 1996; pp 433-436

[24] Pullman S.L., Goodin D.S., Marquinez A.I., Tabbal S., Rubin M., "Clinical utility of surface EMG” Report of the therapeutics and technology assessment subcommittee of the American Academy of Neurology, Neurology, 2000; 55:171-177

[25] Rocon E., Moreno J.C., Ruiz A.F., Brunetti F., Miranda J.A., and Pons J.L., "Application of inertial sensors in rehabilitation robotics”, proceeding of the 2007 IEEE 10th International Conference on Rehabilitation Robotics, June 12-15, 2007, Noordwijk, The Netherlands

[26] Ruth E. Mayagoitaia, Anand V. Nene, Peter H. Veltink (2002) "Accelerometer and rate gyroscope measurement of kinematics: an inexpensive alternative to optical motion analysis systems” Elsevier Journal of Biomechanics, pp. 537-542

[27] Sutliff M.H., "Contribution of impaired mobility to patient burden in multiple sclerosis” Current Medical Research and Opinion, 2010; 26(1):109-119

[28] Winter D.A. (1991) "Biomechanics and Motor control of Human Gait: Normal, Elderly and Pathological”. Waterloo Biomechanics Press. Waterloo, Ontario

[29] World Health Organization, "Neurological Disorders: Public health challenges”, 2006

[30] Wurdeman S.R., Huisinga J.M., Filipi M., and Stergiou N., "Multiple sclerosis affects the frequency content in the vertical ground reaction forces during walking”, Clinical Biomechanics, 2010; 26(2):207-212. 